\title{
Modelling Multi-Vendor Transponders Performance and Optimizing Launch Power
}

\author{
Ankush Mahajan(1), Konstantinos (Kostas) Christodoulopoulos(3), Ricardo Martínez ${ }^{(1)}$, \\ Salvatore Spadaro(2), Raul Muñoz ${ }^{(1)}$
}

(1) Centre Tecnològic de Telecomunicacions de Catalunya, CTTC/CERCA, Castelldefels, 08860, Spain, ankush.mahajan@cttc.cat

(2) Universitat Politècnica de Catalunya (UPC), Barcelona, Spain

(3) Nokia Bell Labs, Stuttgart, Germany

Abstract We propose physical layer model extensions that capture the performance variations of multi-
vendor transponders and use those with appropriate algorithms to optimize connections' launch powers.
We estimated potential SNR improvements upto $\sim 35 \%$ for 4 different vendors in a network of 27 nodes.

\section{Introduction}

The concept of disaggregation of optical transport systems is considered by several operators as a means of higher flexibility and cost reduction. Different levels of disaggregation are being discussed, from partial to full disaggregation with trade-off between data/control plane complexity and cost [1], [2]. Partial disaggregation, where the line system is open (open line system OLS) to multiple vendor transponders (TPs) has gained the most attention due to the ease in the related control plane implementation [1], [3], [4].

In traditional optical transport networks, the line system and the TPs are integrated and under the control of a single vendor (proprietary controller). For such networks, TPs launch power is generally set to a fix value (by the vendor) that is optimized to achieve the best Signal to Noise Ratio (SNR) for the central channel under high load conditions. While optimizing that, several factors need to be accounted, including statistical variations of components of the TPs, even if they come from the same vendor [5]. Used margins, which account for impairment calculation uncertainties and ageing, generally cover such TP performance variations.

For multi-vendor TPs in an OLS, apart from the aforementioned TP statistical variances, vendor dependent factors play a significant role in quality of transmission (QoT)/SNR estimations [4]-[6]. In such a network, performance variations arise from the different TP components and DSP used by the different vendors. So, if we use a typical (single vendor) physical layer model (PLM) [2], [3] we might observe huge deviations in the estimated and measured QoT. Such estimation errors would affect among other operations, the optimization of the launch power levels. In light of the above, herein we propose a scheme to accurately model the physical layer in multi-vendor TP environment that accounts for vendor dependent performance factors. Using this model with appropriate algorithms, we optimize the launch power of connections with the aim to maximize (i). the sum of SNR margins or (ii). the lowest margin for overall network capacity improvement.

\section{Related Work}

Several works study variations of power optimization [7]- [13] with the goal of minimizing intra- and inter-channel non-linear (Kerr) effects since this is the main limiting factor of the transmitted signals power in dispersionuncompensated systems. Previous works proposed heuristics to optimize either the individual [7], [8], [12] [13] or all channel launch power [9]-[11]. Authors in [7], [8] presented several approaches to optimize the launch power (along with the constellation and channel allocations) of each channel to maximize the network efficiency, taking into account nonlinear interference (NLI) as well as amplified spontaneous emission (ASE) noise. The local optimization leads to global optimization (LOGO) model [9] targets on maximizing SNR at the Rx. by maximizing each span's SNR (or minimizing each span's non-linear noise). The main benefit of this model is its flexibility to optimize the power of each span irrespectively of the span lengths (homo or heterogeneous). However, the LOGO assumes full load and the same power levels for all channels (at each span) and thus does not consider the connection distance. Authors in [12] formulated via a convex-based optimization, the problem of individual channel launch powers with the objective of maximizing the minimum SNR margin using a gaussian noise (GN) model. Extensions to take advantage of connections monitoring was presented in [13].

In brief, to the best of our knowledge, the launch power optimization problem in case of partial disaggregation (multi-vendor TPs) has not been investigated. For such networks, a proper PLM is needed to account for vendor dependent TP performance factors. Keeping this in mind, we first propose a PLM to capture the performance of multi-vendor TPs. We then develop methods for optimizing the launch power of each connection based on convex optimization principles. Utilizing our PLM as ground truth, we verify the benefits of our proposed algorithm in maximizing the SNR margins in a multi-vendor TPs environment. 


\section{Multi-Vendor Physical Layer Model}

In single-vendor optical networks, all WDM channels are typically controlled to have uniform launch and span power (by the proprietary controller). The used launch power is typically found to be the optimum with central channel for full load on a multi-span link (Fig. 1(a)). Note that such power optimization results in low efficiency/ excess margins for side channels or diverse network paths. Moreover, the chosen power is typically even more conservative to be on the safe side and ensure the operation of the network in the linear regime. Setting a conservative launch power is definitely a good strategy for networks with limited knowledge about the used elements such as the disaggregated scenario [2].

The GN model [9] is a well-accepted PLM for single- and multi-vendor networks [3],[14]. According to the $\mathrm{GN}$ model (with rectangular signal shape), the impact of transmission effects on the generalized SNR are given by

$S N R=\frac{G_{O}}{G_{A S E}+G_{N L I}}$

where $G_{O}$ is the optical signal average power spectral density (PSD), $G_{A S E}$ is the ASE noise PSD, and $G_{N L I}$ is the NLI noise PSD contribution.
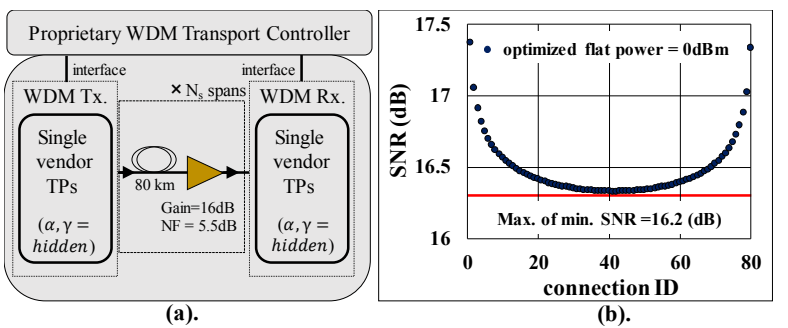

Fig. 1: (a) Traditional WDM transport system: line system and TPs with proprietary controller, (b) SNR (dB) for 80 channels at $32 \mathrm{Gbaud}$ in single link with $\mathrm{N}_{\mathrm{s}}=6$ identical spans

Fig. 1(b) shows the estimated SNR for $80 x$ 32Gbaud pol-mux 16QAM channels, using the traditional PLM of Eq. (1) for a multi-span link (no. of spans, $\mathrm{N}_{\mathrm{s}}=6$ ) and $0 \mathrm{dBm}$ of flat/uniform launch power. We see that the minimum SNR is obtained at the central channel, as expected. We call this as single vendor PLM and denote it by $P L M_{S V}$.

However, the GN model covers impairments, assuming perfect dispersion compensation at the Rx., and does not consider the characteristics of the TPs. In reality $\left\{G_{O}, G_{N L I}\right\}$ terms are affected by TP characteristics such as the DSP implementation, performance variations of TP components (e.g., laser linewidth, photodiode's responsivity, etc.), whereas the linear noise term, $G_{A S E}$, is determined by the optical amplifiers. Based on this, we extended the GN model to capture the TP characteristics in a generic way with the goal to model multi-vendor/ disaggregated scenarios.

In particular, we introduce three performance factors, $\{\alpha, \beta, \gamma\}$, where $\alpha$ covers vendor specific TP components; $\beta$ covers amplifier characteristics; and $\gamma$ covers vendor specific DSP implementation variations [5], [6]. In multi-vendor TPs scenarios, the performance terms $\{\alpha, \beta, \gamma\}$ would be different for the heterogeneous TPs. These should be accounted for in the $P L M_{S V}$ to achieve accurate SNR estimation. Though this model is also applicable for single vendor TPs [6], its importance is more relevant in multi-vendor networks. We consider a scenario, where TPs from $M$ vendors are deployed. For any vendor $i$ out of the $M$, with $\left\{\alpha_{i}, \beta_{i}, \gamma_{i}\right\}$ performance factors, we calculate its SNR with Eq. (2), instead of Eq. (1):

$S N R_{i}=\frac{\alpha_{i} \cdot G_{O}}{\beta_{i} \cdot G_{A S E}+\gamma_{i} \cdot G_{N L I}}=\frac{\left(\alpha_{i} / \beta_{i}\right) \cdot G_{O}}{G_{A S E}+\left(\gamma_{i} / \beta_{i}\right) \cdot G_{N L I}}$
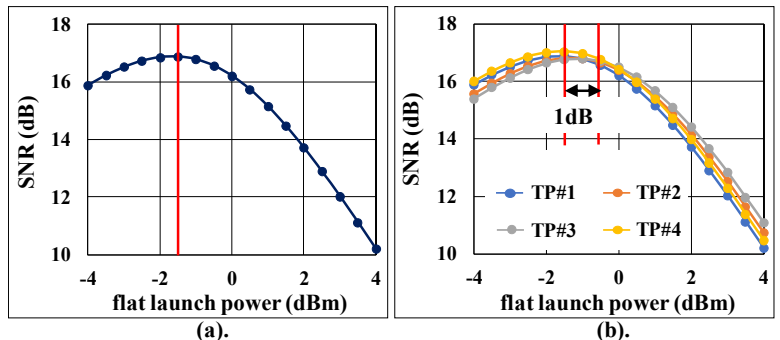

Fig. 2: (a) Estimated $-1.5 \mathrm{dBm}$ (red line) of flat launch power for scenario presented in Fig. 1(a). (b) Optimized flat launch power of central/worst SNR channel for M=4 TP vendors with $\{\alpha, \gamma\}_{\beta=1}$ $=[\{1.0,1.0\},\{0.9,0.8\},\{0.8,0.7\},\{1.0,0.9\}]$ at fixed $\beta=1$.

Eq. (2) accounts for vendor specific performance factors. We call it as multi-vendor PLM and denote it by $P L M_{M V}$. Note that in past, a bias term was added in the accumulation of linear/ASE noise to account for TP implementations [15]. However, our model is quite generic and captures these implementation factors to a great extent, while such a bias term affects the performance, away from our optimization point (in the next section). In the future, such extensions will be considered to further improve the model. Fig.2(a) and 2(b) show the optimized flat launch power for a proprietary network $(\alpha, \beta, \gamma=1)$ and partial disaggregated networks with TPs from $M=4$ different vendors (Fig. 3). In $P L M_{M V}$, we assumed $\beta=1$ since it corresponds to the amplifier performance which even in OLS is under the control of a single vendor. In comparison to Fig. 2(a), we see in Fig. 2(b) a $\sim 1 \mathrm{~dB}$ variation $(-1.5 \mathrm{~dB}$ for TP1, TP4 to $-0.5 \mathrm{~dB}$ for TP2, TP3) of optimal flat launch power for different TPs (resulting in $\sim 0.4 \mathrm{~dB}$ SNR variation, Fig. 3(b)).
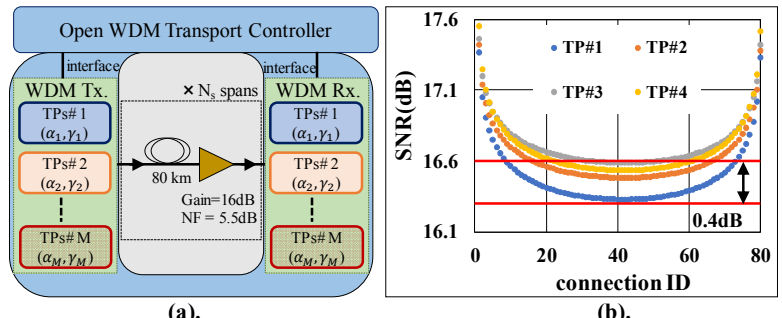

Fig. 3: (a) OLS of multi-vendor TPs with open transport controller, (b) SNR (dB) for 80 channels established with $(M=4)$ TP vendors with $\{\alpha, \gamma\}_{\beta=1}$ similar to Fig. 2(b)

To improve the accuracy of QoT/SNR estimation, we propose to characterize the TPs and identify the vendor specific parameters, or learn them while the network operates [14]. These would be 
used in the $P L M_{M V}$ which in turn would be used in planning or dynamic optimization operations. We now focus on launch power optimization in multivendor TP disaggregated networks.

\section{Launch Power Optimization and Results}

We consider a network with $N$ established connections and two optimization objectives:

i. sum of channel margins:

$$
\max . \sum_{n=1}^{N}\left(\log S N R_{n}(p)-\log S N R_{t h, n}\right)
$$

ii. minimum margin:

subject to:

$$
\max \min _{n \in[1, N]}\left(\log S N R_{n}(p)-\log S N R_{t h, n}\right)
$$

$\log S N R_{n}(p)-\log S N R_{t h, n} \leq 0, \forall n \in[1, N]$

$$
p_{\min } \leq \boldsymbol{p} \leq p_{\max }
$$

where $\boldsymbol{p}=\left[p_{1}, p_{2}, \ldots p_{n}\right]$ is the launch power vector of the $N$ connections; $p_{\min }$ and $p_{\max }$ are the lower and upper power bounds; $S N R_{n}(p)$ is the SNR of connection $n$ at corresponding power vector $p$; and $S N R_{t h, n}$ is the SNR threshold required for the selected modulation format of $n$ [9].

To solve this problem, we firstly implemented a heuristic algorithm. Each connection request consists of a source destination node pair, path/wavelength, mod. format and assigned TP. The heuristic starts from a uniform launch power of $0 \mathrm{dBm}$ and uses $P L M_{M V}$ to estimate SNR value of each connection. It categorizes connections into "low margin" which need better performance, and "high margin" which have sufficient margin w.r.t. the threshold. The heuristic iterates, and in each iteration increases/decreases power of low/high margin connections by a chosen step size $\Delta$.

The optimization of channels' launch powers with the above objectives is known to be convex [12], [13] and of polynomial complexity. Hence, we implemented an interior-point algorithm to solve it. Such algorithm converges faster with a good starting solution. Hence, we used the launch power vector returned by the heuristic as its starting point. Finally, we also applied an adaptive weight neural network (NN) inspired from [16]. The single layer $\mathrm{NN}$ (neurons size $=$ no. of connections) operates with a randomly generated initial population. The best obtained solution at each epoch is set as the target and NN weights are updated accordingly. The NN adapts its weights by moving other predicted solutions towards the target solution, so that the objective improves with iterations.

We considered an Italian backbone topology with 27 nodes and 43 bidirectional links whose lengths range from 8 to $480 \mathrm{~km}$ [11]. We assumed SSMF spans of $80 \mathrm{~km}$, a traffic load of 500 connections with uniformly chosen source-destination nodes, fixed 32 GBaud symbol rate, served in 3 spectrum slots $(37.5 \mathrm{GHz})$. Each demand was modulated at 32Gbaud with PM-\{QPSK, 8-QAM, 16-QAM\} leading to $\{100,150,200\}$ Gbps of data rate. We considered $M=4$ TP vendor classes with $\{\alpha, \gamma\}$ performance factors $[\{1.0,1.0\},\{0.9,0.8\},\{0.8$, $0.7\},\{1.0,0.9\}]$ and fixed $\beta=1$ (Fig. 3). We uniformly
Tab. 1: computation time(sec) for implemented algorithms

\begin{tabular}{|c|c|c|c|c|}
\hline \multirow{2}{*}{$\begin{array}{c}\text { Algorithm } \\
\text { computation time (sec) }\end{array}$} & \multicolumn{2}{|c|}{ Obj.\#1 } & \multicolumn{2}{c|}{ Obj.\#2 } \\
\cline { 2 - 5 } & $\boldsymbol{P L}_{\boldsymbol{S V}}$ & $\boldsymbol{P L}_{\boldsymbol{M V}}$ & $\boldsymbol{P L}_{\boldsymbol{S V}}$ & $\boldsymbol{P L}_{\boldsymbol{M V}}$ \\
\hline heuristic, $\Delta= \pm 1 \mathrm{dBm}$ & 56.5 & 68.8 & 69.5 & 72.4 \\
\hline heuristic, $\Delta= \pm 0.5 \mathrm{dBm}$ & 95.1 & 99.3 & 104.2 & 107.9 \\
\hline heuristic, $\Delta= \pm 0.25 \mathrm{dBm}$ & 120.2 & 131.6 & 134.8 & 147.2 \\
\hline convex+ heuristic & 247.9 & 242.5 & 241.9 & 257.8 \\
\hline NN & 421.7 & 400.4 & 483.6 & 493.9 \\
\hline
\end{tabular}

assigned one TP/modulation to each demand. Finally, we averaged the results over 20 iterations. Tab. 1 shows the computation time of the examined algorithms (heuristic, convex, NN) and PLMs $\left(P L M_{S V}, P L M_{M V}\right)$, while Fig. 4 the objectives performance. We observe a trade-off between computation time and objectives. The performance of the heuristic varies for the chosen step size $\Delta$, the objective is better for smaller $\Delta$, while its computation time increases almost linearly with $\Delta$.

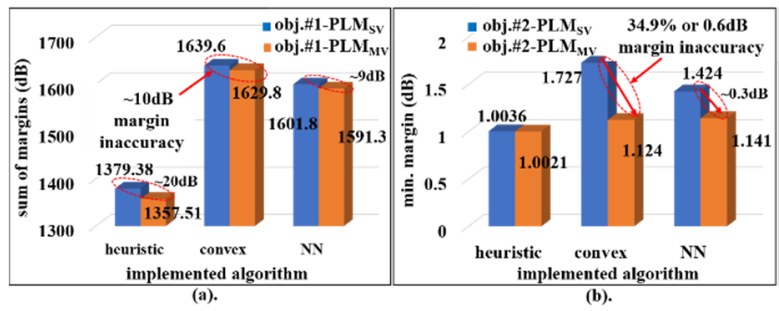

Fig. 4: (a) obj.\#1 (b) obj.\#2 values and related savings when using $P L M_{M V}$ instead of $P L M_{S V}$ for the multi-vendor scenario

The NN algorithm achieved good performance (better than the heuristic) but with the highest execution time (even higher than the convex). NN performance is generally quite good for finding non-linear mapping functions. However, for the multi-vendor launch power optimization problem at hand, convex optimization performed much better (Fig. 4). The convex (interior point) algorithm achieved the best performance in reasonable/low time. We observed $\sim 10 \mathrm{~dB}$ of obj.\#1 improvement and $\sim 0.6 \mathrm{~dB}$ (34.9\%) improvement of obj.\#2 when using $P L M_{M V}$ instead of $P L M_{S V}$ for the convex algorithm in the studied multi-vendor scenario. This improvement comes from the inaccuracy of the $P L M_{S V}$. Note that the minimum margin (obj.\#2) reflects connections' infeasibility. Hence the use of proposed $P L M_{M V}$ reduces substantially the infeasibility probability of connections in a multivendor network. Varying improvements, depending on algorithm's performance and PLM inaccuracy were observed for the other examined algorithms (Fig. 4).

\section{Conclusion}

We proposed a TP vendor dependent physical layer model for more accurate SNR estimation. Using this multi-vendor PLM with convex algorithms, we observed $\sim 0.6 \mathrm{~dB}$ higher SNR estimation accuracy and $\sim 35 \%$ min. margin savings compared to a traditional PLM model.

\section{Acknowledgements}

This work is a part of ONFIRE project supported by EU Horizon 2020 research and innovation programme under the MSCA grant agreement No. 765275. 


\section{References}

[1] E. Riccardi et al., "An Operator view on the Introduction of White Boxes into Optical Networks", IEEE JLT, vol. 36, no. 15, pp. 3062-3072, 2018

[2] J. Santos et al., "On the Impact of Deploying Optical Transport Networks Using Disaggregated Line Systems", IEEE/OSA JOCN, vol. 10, no. 1, pp. A60-A68, 2018

[3] M. Filer et al., "Multi-vendor Experimental Validation of an Open Source QoT Estimator for Optical Networks", IEEE JLT, vol. 36, no. 15, pp. 3073-3082, 2018

[4] M. P. Belanger et al., "Margin Requirement of Disaggregating the DWDM Transport System and its Consequence on Application Economics", OFC, San Diego, California, USA, 2018

[5] "Evolving the Awareness of Optical Networks", whitepaper, Infinera

[6] Y. R. Zhou et al., "Demonstration of a Novel Technique for Non-Intrusive In-Band OSNR Derivation Using Flexible Rate Optical Transponders Over a Live $727 \mathrm{~km}$ Flexible Grid Optical Link", IEEE JLT, vol. 35, no. 20, pp. 43994405, 2017

[7] D. J. Ives et al., "Transmitter Optimized Optical Networks", OFC/NFOEC, Anaheim, California, USA, 2013

[8] D. J. Ives et al., "Adapting Transmitter Power and Modulation Format to Improve Optical Network Performance Utilizing the Gaussian Noise Model of Nonlinear Impairments", IEEE JLT, vol. 32, no. 21, pp. 3485-3494, 2014

[9] P. Poggiolini et al., "The GN-Model of Fiber Non-Linear Propagation and its Applications", IEEE JLT, vol. 32, no. 4, pp. 694-721, 2013

[10] L. Yan et al., "Power Optimization in Nonlinear FlexibleGrid Optical Networks", ECOC, Valencia, Spain, 2015

[11] P. Soumplis et al., "Network Planning with Actual Margins", IEEE JLT, vol. 35, no. 23, pp. 5105-5120, 2017

[12] I. Roberts et al., "Convex Channel Power Optimization in Nonlinear WDM Systems using Gaussian Noise Model", IEEE JLT, vol. 34, no. 13, pp. 3212-3222, 2016

[13] I. Roberts et al., "Measurement-Based Optimization of Channel Powers with Non-Gaussian Nonlinear Interference Noise", IEEE JLT, vol. 36, no. 13, pp. 27462456, 2018

[14] A. Mahajan et al., "Modeling EDFA Gain Ripple and Filter Penalties with Machine Learning for Accurate QoT Estimation", IEEE JLT, vol. 38, no. 9, pp. 2616-2629, 2020

[15] F. Vacondio et al., "On Nonlinear Distortions of Highly Dispersive Optical Coherent Systems", Optics Express, vol. 20, no. 2, pp. 1022-1032, 2012

[16] A. Sadollah et al., "A Dynamic Metaheuristic Optimization Model Inspired by Biological Nervous Systems: Neural Network Algorithm", Elsevier, ASC, vol. 71, pp. 747-782, 2018 